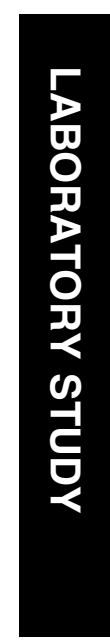

\title{
Short-term effect of intracameral triamcinolone acetonide on corneal endothelium using the rabbit model
}

${ }^{1}$ Department of

Ophthalmology, Seoul

National University College of Medicine, Seoul Artificial Eye Center, Seoul National University Hospital Clinical Research Institute, Seoul, Korea

${ }^{2}$ Seoul National University, Bundang Hospital, Seongnam, Korea

Correspondence: MK Kim, Department of Ophthalmology, Seoul National University College of Medicine, 28 Yongondong, Chongno-gu, Seoul 110-744, Korea

Tel.: + 8222072 2665;

Fax: + 8227413187.

E-mail:kmk9@

medimail.co.kr

Received: 17 November 2005

Accepted in revised form: 16 March 2006

Published online: 2 June 2006

None of the authors has a financial or proprietary interest in any of the material or method mentioned

Presented in part at the ASCRS Symposium, Washington DC, USA, April 2005
Abstract

Purpose To investigate the effect of intracameral injection of triamcinolone acetonide on the corneal endothelium in rabbit eyes.

Methods Triamcinolone acetonide $(40 \mathrm{mg} / \mathrm{ml}$, $0.2 \mathrm{~cm}^{3}$ ) after filtering and resuspension in balanced salt solution (BSS) was injected intracamerally for 3 min into 10 rabbit eyes and irrigated with $5 \mathrm{~cm}^{3}$ of BSS. Triamcinolone without resuspension and BSS were injected, respectively, into five rabbit eyes. Endothelial toxicity was evaluated and compared by measurements of endothelial cell counts and central corneal thickness. The endothelial viability was determined using vital staining with alizarin red and trypan blue at $2 \mathrm{~h}$ after injection. The scanning electron microscopy (SEM) was performed in one cornea from each group.

Results Endothelial cell counts and central corneal thickness following intracameral injection of triamcinolone acetonide did not significantly change when compared to controls. The mean percentage of viable endothelial cells was 99.50, 99.52, and $99.49 \%$ in the resuspended triamcinolone group, triamcinolone without resuspension group, and BSS group, respectively $(P=0.46$, Kruskall-Wallis test). But SEM showed reduced microvilli of endothelial surface in an eye of the triamcinolone without resuspension group.

Conclusions The intracameral injection of triamcinolone acetonide did not induce a significant visable change of endothelium in rabbit eyes. However, ultrastructural villi changes observed suggest a possibility of microstructural damages in endothelium with
JY Oh'1, WR Wee'2, JH Lee ${ }^{1}$ and MK Kim¹

triamcinolone acetonide injection when used without filtering and resuspension.

Eye (2007) 21, 812-818; doi:10.1038/sj.eye.6702357; published online 2 June 2006

Keywords: cornea; effect; endothelium; toxicity; triamcinolone

\section{Introduction}

Triamcinolone acetonide is being used increasingly to enhance visualization of the vitreous body in the anterior chamber of the eye during cataract surgery. ${ }^{1-3}$ Enhanced viewing of these tissues can promote a surgeon's ability to evaluate clinical structural relationships and may aid in a more effective anterior vitrectomy with fewer complication. In addition, there are reports suggesting that intracameral injection of dexamethasone during cataract surgery reduces the postoperative inflammation ${ }^{2}$ and subsequently the development of postoperative cystoid macular oedema. However, corneal endothelial toxicity with the intracameral injection of triamcinolone acetonide has not been fully evaluated to date. The preparation of triamcinolone acetonide includes preservatives such as benzyl alcohol. Therefore, we would predict that some endothelial damage can result from triamcinolone acetonide or preservatives. We investigated the short-term effect of intracameral triamcinolone acetonide on the corneal endothelium using a rabbit model.

\section{Materials and methods}

Animal experiments were performed in accordance with the Association for Research 
in Vision and Ophthalmology Statement for Use of Animals in Ophthalmic Vision and Research, and the protocol was approved by the Institutional Review Board of Seoul National University Hospital, Seoul, Korea.

Twenty New Zealand white rabbits were randomly divided into three groups and designated as resuspended triamcinolone group, triamcinolone without resuspension group, and BSS group as a control. Ten rabbits in the resuspended triamcinolone group received an intracameral injection of triamcinolone acetonide (Tamceton ${ }^{\circledR}$, Hanall Pharmaceutical, Seoul, Korea; $40 \mathrm{mg} / \mathrm{ml}, 0.2 \mathrm{~cm}^{3}$ ) resuspended in balanced salt solution (BSS, Santen, Osaka, Japan) to avoid injecting a vehicle present in commercially available triamcinolone acetonide; the triamcinolone acetonide solution was made by the method that a triamcinolone aqueous suspension, was left standing for $30 \mathrm{~min}$ and the vehicle was discarded, as previously described ${ }^{4}$ and the remaining triamcinolone acetonide suspension was washed with $1 \mathrm{ml}$ of BSS three times using a $0.2 \mu \mathrm{m}$ filter (Millex-FG ${ }^{\circledR}$, Carringtwohill, Co., Cork, Ireland) to thoroughly remove a vehicle possibly remaining in the suspension. Five rabbits were included in the triamcinolone without resuspension group, they received an injection of commercial preparation of triamcinolone acetonide without filtering or resuspension $\left(40 \mathrm{mg} / \mathrm{ml}, 0.2 \mathrm{~cm}^{3}\right)$. The third group, a control group, had five rabbits, they received an intracameral injection of BSS.

Surgery was performed randomly on one eye of a rabbit. The rabbits were anaesthetized with an intramuscular injection of ketamine $(30 \mathrm{mg} / \mathrm{kg})$ and xylazine $(5 \mathrm{mg} / \mathrm{kg})$. A wire lid speculum was placed to separate the eyelids. An operating microscope was positioned over the eye undergoing surgery. The anterior chamber was entered in the superotemporal quadrant through a long corneal tunnel using a 30-G needle. After entry, $0.2 \mathrm{ml}$ of aqueous humor was withdrawn from the eye and then $0.2 \mathrm{ml}$ of triamcinolone acetonide or BSS was injected intracamerally through a separate $30-\mathrm{G}$ needle. Three minutes later, the anterior chamber was irrigated with $5 \mathrm{ml}$ of BSS by the bimanual method to wash away the triamcinolone.

Corneal thickness measurements by ultrasonic pachymetry (Pachymeter echograph, Quantel medical, Clermont-Ferrand, France) and endothelial cell count by specular microscopy (Konan specular microscope SP8800, Konan medical Inc., Nishinomiya, Japan) were made before surgery and every $30 \mathrm{~min}$ after surgery. Within each group, temporal changes in corneal thickness and endothelial cell counts were tested using a paired Student's $t$-test. The difference between baseline and postoperative measurements in corneal thickness and endothelial cell counts were obtained by subtracting baseline measurements from measurements at $2 \mathrm{~h}$ after injection in each group and the mean change was compared between the three groups using Kruskal-Wallis test.

Two hours after the injection, the rabbits were killed with an intracardiac injection of potassium chloride. Their corneas and a 2.0-mm rim of sclera were removed with a blade and scissors and the iris diaphragm was peeled from the cornea. In 17 of a total of 20 corneas (nine from the resuspended triamcinolone group, four from the triamcinolone without resuspension group, and four from the BSS group), the endothelial viability was determined using a live/dead cell assay according to Taylor and Hunt. ${ }^{5}$ Corneal buttons were placed endothelial side up and $0.25 \%$ trypan blue was applied dropwise to the endothelium for $90 \mathrm{~s}$. Each cornea was rinsed with normal saline to remove excess stain. Alizarin red S (0.20\%; pH 4.2) was then applied to the endothelium for $90 \mathrm{~s}$ and the buttons were rinsed with normal saline. After the staining procedure, corneas were immersed in the glutaraldehyde fixative solution (2.48\%; osmolality $301 \mathrm{mosm} / \mathrm{kg} \mathrm{pH} \mathrm{7.2)} \mathrm{for} 10 \mathrm{~min}$. Each cornea was prepared by trephining in a centre with a $6 \mathrm{~mm}$ of trephine (Storz Ophthalmics, St Louis, MI, USA) to reduce biased information about endothelial cell damages derived from the cutting with scissors during the harvest of the cornea. The disc of tissue was mounted, endothelium uppermost, in saline, under a coverslip, on a glass microscope slide and was then evaluated for endothelial damage with an Olympus BX 50 light microscope (Olympus Optical Co. Ltd., Tokyo, Japan). The number of trypan blue-stained dead cells and unstained live cells was counted in a more than 10 medium power field $(\times 200)$ using a grid in the microscope to calculate the percentages of dead cells. The mean percentage of dead endothelial cells was compared between the three groups using Kruskal-Wallis test.

The remaining three corneas, one cornea from each group, were dissected in piece, $3 \times 3 \mathrm{~mm}$ from the centre and prepared for scanning electron microscopy (SEM). Samples were washed in sodium buffer solution (PBS) and cut into smaller pieces. For SEM, samples were prefixed with $2.5 \%$ glutaraldehyde PBS ( $\mathrm{pH} 7.2$ ) at $4{ }^{\circ} \mathrm{C}$ overnight. Following several washes in PBS, samples were kept in $1 \%$ osmiumtetraoxide-PBS for the final fixation for $1 \mathrm{~h}$. Samples were then washed and dehydrated through serial dilutions of ethanol. Samples were mounted onto stubs, sputter-coated with gold by Polaron SC-500 (VG Microtech, Sussex, UK) and finally examined with SEM (JSM 300 JEOL, Tokyo, Japan). 


\section{Results}

\section{Corneal thickness}

In the resuspended triamcinolone group, the mean central corneal thickness $( \pm S D)$ was $359 \pm 32 \mu \mathrm{m}$ at baseline and $335 \pm 24,330 \pm 19$, and $327 \pm 24 \mu \mathrm{m}$ at $0.5,1$, and $2 \mathrm{~h}$ after injection, respectively. In the triamcinolone without resuspension group, the mean corneal thickness $( \pm \mathrm{SD})$ was $380 \pm 28 \mu \mathrm{m}$ at baseline and $360 \pm 16,384 \pm 32$, and $390 \pm 35 \mu \mathrm{m}$ at $0.5,1$, and $2 \mathrm{~h}$ after injection, respectively. In the BSS group, the mean corneal thickness $( \pm \mathrm{SD})$ was $368 \pm 24 \mu \mathrm{m}$ at baseline and $375 \pm 25,370 \pm 20$, and $376 \pm 19 \mu \mathrm{m}$ at $0.5,1$, and $2 \mathrm{~h}$ after injection, respectively. In each group, central corneal thickness was not significantly different from baseline at $2 \mathrm{~h}$ after injection $(P=0.241$ in suspended triamcinolone group, $P=0.531$ in triamcinolone without resuspension group, and $P=0.541$ in BSS group) (Figure 1). After comparison of the three groups, the change in central corneal thickness after injection was not significant. The mean change between baseline values and at postoperative $2 \mathrm{~h}$ was $-25.5 \pm 24.1 \mu \mathrm{m}$ in suspended triamcinolone group, $+13.5 \pm 20.0 \mu \mathrm{m}$ in triamcinolone without resuspension group, and $+11.0 \pm 17.3 \mu \mathrm{m}$ in BSS group ( + means an increase in corneal thickness; - means a decrease in corneal thickness). No one group experienced a change greater than the others $(P=0.418$; this $P$-value encompasses all three groups).

\section{Endothelial cell counts}

The mean endothelial cell count $( \pm S D)$ was $2902 \pm 129$ at baseline and $2930 \pm 141,2913 \pm 151$, and $2922 \pm 124$ at $0.5,1$, and $2 \mathrm{~h}$ after injection, respectively, in the suspended triamcinolone group. In the triamcinolone without resuspension group, the mean endothelial cell count $( \pm S D)$ was $2883 \pm 109$ at baseline and $2893 \pm 116,2886 \pm 122$, and $2873 \pm 107$ at $0.5,1$, and $2 \mathrm{~h}$ after injection, respectively. In the BSS group, the mean endothelial cell count was $2945 \pm 106$ at baseline and $2921 \pm 105,2949 \pm 110$, and $2900 \pm 109$ at 0.5 , 1 , and $2 \mathrm{~h}$ after injection, respectively. The mean endothelial cell count was not significantly different from baseline when compared to $2 \mathrm{~h}$ after injection in each group studied ( $P=0.413$ in the suspended triamcinolone group, $P=0.395$ in the triamcinolone without resuspension group, and $P=0.627$ in the BSS group) (Figure 2). The mean change between baseline and measurements at postoperative $2 \mathrm{~h}$ was $+21.0 \pm 27.7$ in the suspended triamcinolone group, $-11.3 \pm 22.8$ in the triamcinolone without resuspension group, and $-40.0 \pm 18.1$ in the BSS group, respectively ( + means an increase in endothelial cell count; - means a decrease in endothelial cell count). There were no significant difference observed between the three groups studied ( $P=0.502$; this $P$-value encompasses all three groups).

\section{Live/dead cell assay}

The vital stain revealed that the mean percentage of dead endothelial cells $( \pm S D)$ was $0.50 \pm 0.23 \%, 0.48 \pm 0.20 \%$,

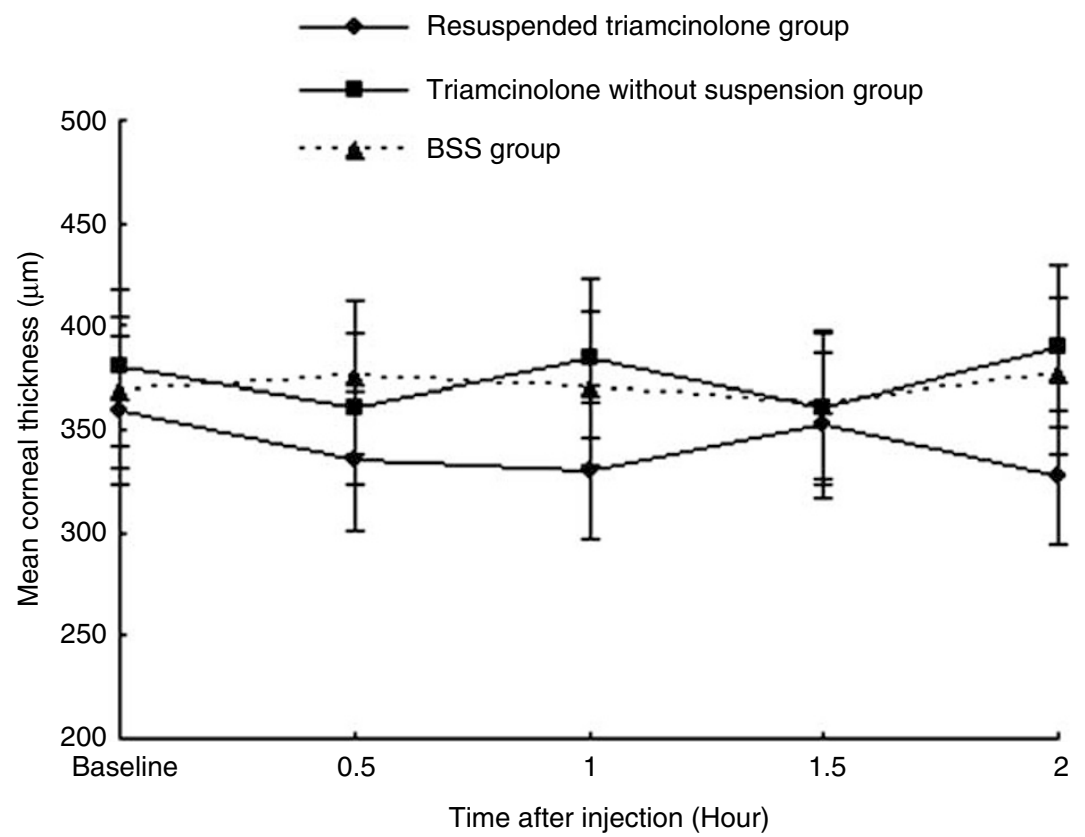

Figure 1 Temporal change, in the mean central corneal thickness, from baseline to the time after anterior chamber injection, in the three groups. The mean central corneal thickness was not significantly different, from baseline and at $2 \mathrm{~h}$ after injection, for each group. 


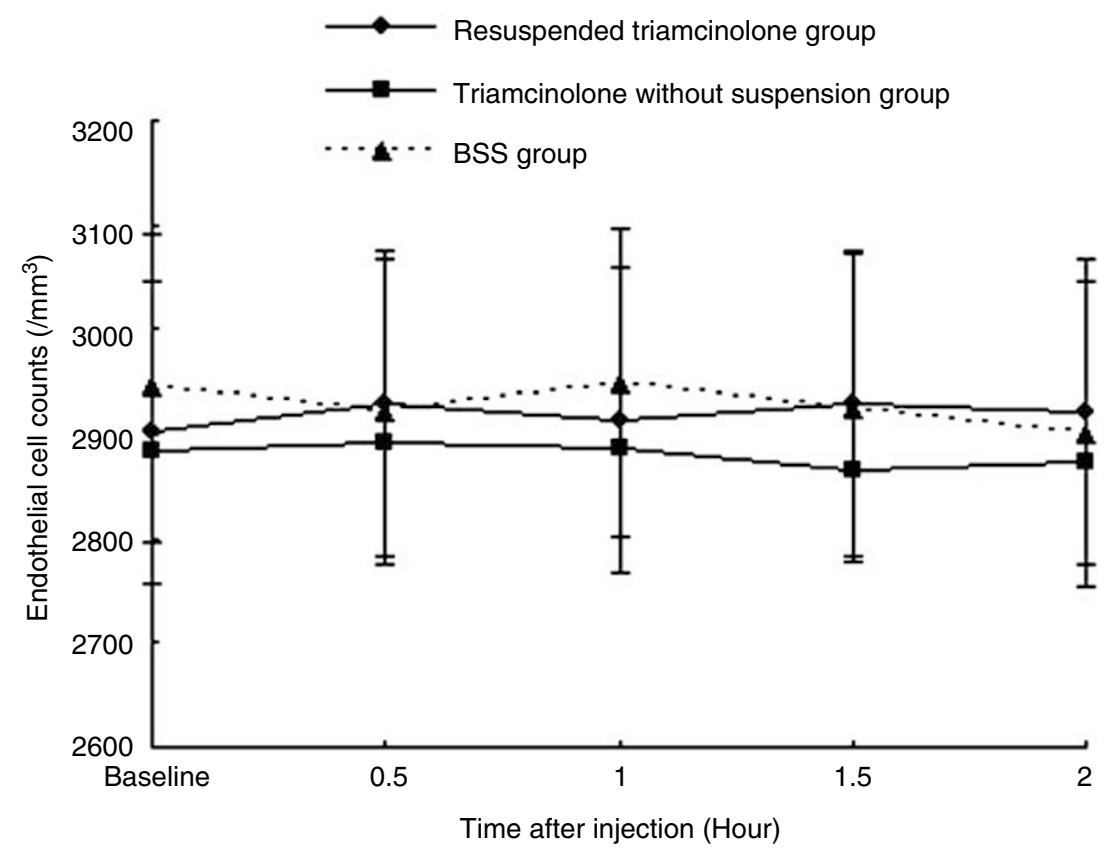

Figure 2 Temporal change in the mean endothelial cell count, from baseline to the time after the anterior chamber injection, in the three groups. The mean endothelial cell count was not significantly changed, from baseline and at $2 \mathrm{~h}$ after injection, for each group.

and $0.51 \pm 0.15 \%$ in the suspended triamcinolone group, in the triamcinolone without resuspension group, and in the BSS group, respectively. No significant differences were noted $(P=0.460$; this $P$-value encompasses all three groups). Therefore, intracameral injection of triamcinolone acetonide did not cause significant endothelial death compared with that in the BSS-injected eyes (Figure $3 a-c$ ).

\section{Electron microscopy}

SEM revealed that endothelial cells kept hexagonality and presented well-defined cell borders in all three groups regardless of whether they were the triamcinolone-injected corneas or the controls. However, microvilli of the endothelial surface were reduced in number in the triamcinolone without resuspension group, whereas microvilli were well maintained in the resuspended triamcinolone group and the BSS group (Figure $4 \mathrm{a}-\mathrm{c}$ ).

\section{Discussion}

Triamcinolone acetonide is a water-insoluble steroid which inhibits inflammation and has been used as a potent anti-inflammatory agent for various intraocular diseases. Recently, Yamakiri et $a l^{1}$ reported that the intracameral injection of triamcinolone acetonide during cataract surgery visualizes the transparent vitreous in the anterior chamber and that this method helps surgeons to remove the vitreous body completely and safely from the anterior chamber. Nonetheless, there are several concerns related to the use of triamcinolone acetonide intraocularly including intraocular pressure elevation, ${ }^{1}$ postoperative endophthalmitis, ${ }^{2,6}$ and corneal endothelial toxicity. Regarding corneal toxicity, Chan et $a l^{7}$ reported that a single $4 \mathrm{mg}$ bolus injection of intravitreal triamcinolone acetonide had no harmful effects on the human corneal endothelium during 6 months of follow-up, when evaluated with specular microscopy. But there has been no prior report evaluating endothelial effect of intracameral triamcinolone to our knowledge. Therefore, we evaluated the effect on corneal endothelium of rabbit eyes when exposed to triamcinolone acetonide for $3 \mathrm{~min}$ after intracameral injection.

In our study, 3-min exposure to $8 \mathrm{mg} / 0.2 \mathrm{ml}$ of triamcinolone acetonide did not produce significant changes in corneal thickness and endothelial cell counts compared to those before injection and those in control groups. A quantatitive live/dead cell assay showed that endothelial cell viability was not altered after a 3-min exposure to triamcinolone acetonide. These findings suggest that short-term injection of triamcinolone with or without resuspension might not affect the viability of the corneal endothelial cells. SEM revealed that microvilli slightly decreased in number in the triamcinolone without resuspension group, suggesting a possible microstructural damage. However, endothelial cells were 

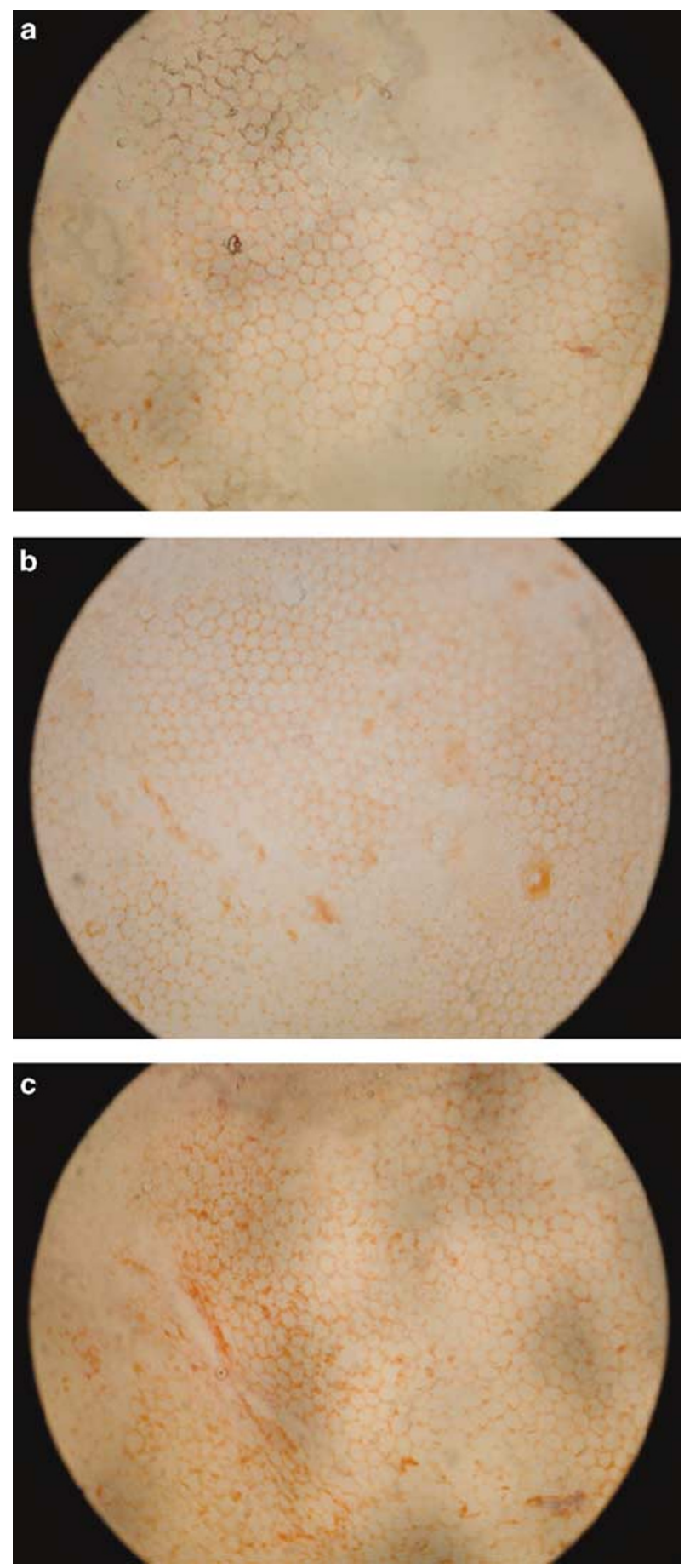

Figure 3 Light microscopic view of rabbit corneas stained with trypan blue and alizarin red after $3 \mathrm{~min}$ of exposure to a commercial preparation of triamcinolone acetonide without resuspension $(40 \mathrm{mg} / \mathrm{ml})$ (a), triamcinolone acetonide after filtered and resuspended $(40 \mathrm{mg} / \mathrm{ml})$ (b) and BSS (c). The endothelial cells in all three groups had a regular hexagonal shape with straight, well-demarcated borders (original magnification $\times 400$ ).
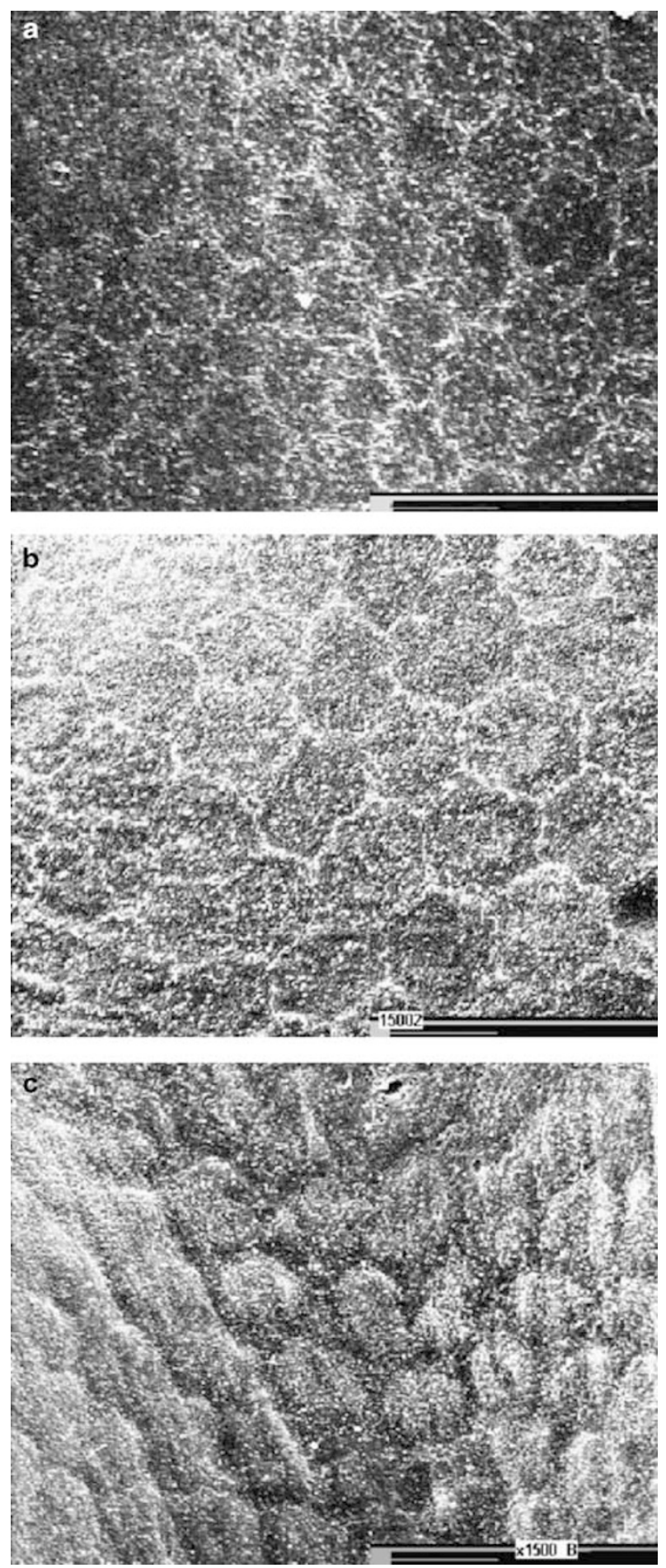

Figure 4 Scanning electron micrographs of the endothelium of rabbit corneas after $3 \mathrm{~min}$ of exposure to commercial preparation of triamcinolone acetonide without resuspension $(40 \mathrm{mg} / \mathrm{ml})$ (a), triamcinolone acetonide after filtered and resuspended (b), and BSS (c). The endothelium in triamcinolone without resuspension group (a) showed a loss of microvilli whereas the endothelium in the resuspended triamcinolone group (b) and BSS group (c) revealed normal microvilli on the surface (original magnification $\times 1500$ ). 
found to keep hexagonality and well-defined cell borders in all the three groups.

The preservatives in the preparation of triamcinolone $(40 \mathrm{mg} / 1 \mathrm{ml})$ include: benzyl alcohol $0.045 \%$ and other components such as sodium carboxymethyl cellulose $2 \mathrm{mg}$, polysorbate $2 \mathrm{mg}$, sodium chloride $9 \mathrm{mg}$ and sodium hydroxide. Reduction of microvilli might be owing to corneal cytotoxicity of benzyl alcohol or other additives like carboxymethyl cellulose because microvilli were maintained in eyes injected with triamcinolone acetonide after being washed and resuspended in BSS. Also, there is a report demonstrating that toxic effects of triamcinolone on the retina could be caused by preservatives alone or by the osmolarity of the vehicles, of various commercially available corticosteroids, when injected intravitreally in rabbit eyes. ${ }^{8}$

The cytotoxicity of triamcinolone has been reported in in vitro cultures. ${ }^{9}$ In a report of a 5-day exposure of triamcinolone acetonide including benzyl alcohol $0.025 \%$, the numbers of ARPE19 cells were reduced significantly. The threshold of resistance against the triamcinolone preparation may be different between retinal pigment epithelium cells and corneal endothelial cells. The exposure time is an essential factor when cell damage is considered. Therefore, in vitro outcome with long-term exposure in the above study, might be expected to have different results when compared to our in vivo data that show no changes in viability except a slight microstructural damage after short-term exposure. We believe our model has more relevance to clinical cataract surgery because anterior chamber was irrigated with BSS soon after injection of triamcinolone in cataract surgery.

The present study has several limitations. First, unlike the human counterpart, rabbit corneal endothelium maintains mitotic activity and regenerates after thermal and chemical insult. ${ }^{10,11}$ There are better models including the cat ${ }^{10}$ and monkey ${ }^{12}$ eye where mitotic activity is rare. We used the rabbit model for pragmatic reasons including the lower cost associated with maintaining rabbits, the ease of obtaining pachymetry measurements and corneal photographs, the morphological similarities between rabbit and human corneas, and the general familiarity of the medical community with the rabbit model. To reduce the bias from the proliferative potential in the rabbit, we examined the cells $2 \mathrm{~h}$ after exposure, for which corneal endothelial cells could not regenerate. ${ }^{13-16}$ Second, we did not evaluate the endothelial change when the cornea was exposed to triamcinolone acetonide for more than $3 \mathrm{~min}$. A long-term exposure study is needed to evaluate cytotoxicity during surgery. Third, the cell viability was examined $2 \mathrm{~h}$ after injection. Actually, we preliminarily checked the viability up to $12 \mathrm{~h}$, and we did not find any difference of viability till $12 \mathrm{~h}$. Cell death like apoptosis is known to appear high $2 \mathrm{~h}$ after exposure of certain damage. ${ }^{13}$ Moreover, rabbit endothelial cells can be regenerated after damage. Therefore, we chose $2 \mathrm{~h}$ after injection as an examination time to decrease bias related to endothelial proliferation although possibility that cell damage took longer time than $2 \mathrm{~h}$ might not be excluded.

In conclusion, our study showed that a 3-min exposure to triamcinolone acetonide $(40 \mathrm{mg} / \mathrm{ml})$ had no toxic effect on the endothelial function and viability in rabbit cornea. However, changes observed in ultrastructural villi suggest a possible microstructural damage to endothelium after exposure to commercial out of the bottle triamcinolone acetonide without resuspension. Therefore, cautious use of the intracameral injection of triamcinolone, after washing and resuspension in BSS, is recommended.

\section{References}

1 Yamakiri K, Uchino E, Kimura K, Azad RV. Intracameral triamcinolone helps to visualize and remove the vitreous body in anterior chamber in cataract surgery. $A m \mathrm{~J}$ Ophthalmol 2004; 138: 650-652.

2 Tan DT, Chee SP, Lim L, Theng J, Van Ede M. Randomized clinical trial of Surodex steroid drug delivery system for cataract surgery anterior versus posterior placement of two Surodex in the eye. Ophthalmology 2001; 108: 2172-2181.

3 Burk SE, Da Mata AP, Snyder ME, Schneider S, Osher RH, Cionni RJ. Visualizing vitreous using Kenalog suspension. J Cataract Refract Surg 2003; 29: 645-651.

4 Peyman GA, Cheema R, Conway MD, Fang T. Triamcinolone acetonide as an aid to visualization of the vitreous and the posterior hyaloid during pars plana vitrectomy. Retina 2000; 20: 554-555.

5 Taylor MJ, Hunt CJ. Dual staining of corneal endothelium with trypan blue and alizarin red S: importance of $\mathrm{pH}$ for the dye-lake reaction. Br J Ophthalmol 1981; 65: 815-819.

6 Sakamoto T, Enaida H, Kubota T, Nakahara M, Yamakiri K, Yamashita $\mathrm{T}$ et al. Incidence of acute endophthalmitis after triamcinolone-assisted pars plana vitrectomy. Am J Ophthalmol 2004; 138: 137-138.

7 Chan CK, Fan DS, Chan WM, Lai WW, Lee VY, Lam DS. Ocular-hypertensive response and corneal endothelial changes after intravitreal triamcinolone injections in Chinese subjects: a 6-month follow-up study. Eye 2005; 19: 625-630.

8 Hida T, Chandler D, Arena JE, Machemer R. Experimental and clinical observations of the intraocular toxicity of commercial corticosteroid preparations. Am J Ophthalmol 1986; 101: 190-195.

9 Yeung CK, Chan KP, Chiang SW, Pang CP, Lam DS. The toxic and stress responses of cultured human retinal pigment epithelium (ARPE19) and human glial cells (SVG) in the presence of triamcinolone. Invest Ophthalmol Vis Sci 2003; 44: 5293-5300.

10 Van Horn DL, Sendele DD, Seideman S, Buco PJ. Regenerative capacity of the corneal endothelium 
in rabbit and cat. Invest Ophthalmol Vis Sci 1977l; 16: 597-613.

11 Olsen EG, Davanger M. The healing of rabbit corneal endothelium. Acta Ophthalmol 1984; 62: 796-807.

12 Van Horn DL, Hyndiuk RA. Endothelial wound repair in primate cornea. Exp Eye Res 1975; 21: 113-124.

13 Holly GP, Alam A, Kiri A, Edelhauser HF. Effect of indocyanine green intraocular stain on human and rabbit corneal endothelial structure and viability. An in vitro study. J Cataract Refract Surg 2002; 28: 1027-1033.
14 Judge AJ, Najafi K, Lee DA, Miller KM. Corneal endothelial toxicity of topical anesthesia. Ophthalmology 1997; 104: 1373-1379.

15 Li Y, Feng G, Yi Y, Zhong X, Zheng H. Observation of the endothelial healing in rabbit corneal alkali wounds by alizarin red S-trypan blue staining method. Yan Ke Xue Bao 1999; 15: 218-220.

16 Medin W, Davanger M. Toxic effects of ouabain on the rabbit corneal endothelium. Acta Ophthalmol (Copenh) 1993; 71: 365-370. 